\title{
Erratum to: Beak colour reflects circulating carotenoid and vitamin A levels in spotless starlings (Sturnus unicolor)
}

\author{
Carlos Navarro • Tomás Pérez-Contreras • \\ Jesús M. Avilés • Kevin J. McGraw • Juan J. Soler
}

Published online: 18 May 2010

(C) Springer-Verlag 2010

\section{Erratum to: Behav Ecol Sociobiol \\ DOI 10.1007/s00265-010-0920-5}

The original version of this article unfortunately contained numerous spelling and grammatical mistakes, but also the following factual errors that we wish to correct here:

1) The surname of one of the authors was misspelled. Instead "Kevin J Mcgraw" should read "Kevin J McGraw".

2) The journal for one of the citations in the reference list was incorrectly given. Here is the corrected entry:
"Saks L, McGraw K, Horak P (2003) How feather colour reflects its carotenoid content. Funct Ecol 17:555-561". 3 ) In the legend for Tables 2 and 3, "total carotenoid plasma level (TCPL)" should read "total plasma carotenoid level (TPCL)". Also in Table 2, "Date2" should be "Date".

4) Throughout the Methods and Results sections, periods were used to separate the degrees of freedom for the numerator and denominator of $F$ statistics; instead, these should be separated by commas.

5) In the 6th line of the 2nd paragraph of the Discussion section, "plasma-serum ratio" should be replaced with "plasma/red blood cells ratio".

The online version of the original article can be found at http://dx.doi. org/10.1007/s00265-010-0920-5.

C. Navarro $(\bowtie) \cdot$ T. Pérez-Contreras $\cdot$ J. M. Avilés $\cdot J$ J. J. Soler

Departamento de Ecología Funcional y Evolutiva,

Estación Experimental de Zonas Áridas (CSIC),

E-04001 Almería, Spain

e-mail: cnavarro@eeza.csic.es

J. M. Avilés

Departamento de Biología Animal y Ecología,

Universidad de Granada,

E-18071 Granada, Spain

K. J. McGraw

School of Life Sciences, Arizona State University,

Tempe, AZ 85287-4501, USA 